\title{
A Case Series of 12 Patients Receiving Bilateral Transverse Abdominis Plane Blocks after Cesarean Section
}

\author{
Dennis Feierman, Aden Bronstein, Miriam Flaum, Marc Dany, Mohit Garg, Piyush Gupta, \\ Kalpana Tyagaraj
}

Department of Anesthesiology, Maimonides Medical Center, Brooklyn, NY, USA

Email: dfeierman@maimonidesmed.org

How to cite this paper: Feierman, D., Bronstein, A., Flaum, M., Dany, M., Garg, M., Gupta, P. and Tyagaraj, K. (2017) A Case Series of 12 Patients Receiving Bilateral Transverse Abdominis Plane Blocks after Cesarean Section. Open Journal of Anesthesiology, 7, 407-414.

https://doi.org/10.4236/ojanes.2017.712042

Received: November 28, 2017

Accepted: December 25, 2017

Published: December 28, 2017

Copyright $\odot 2017$ by authors and Scientific Research Publishing Inc. This work is licensed under the Creative Commons Attribution International License (CC BY 4.0).

http://creativecommons.org/licenses/by/4.0/ Open Access

\begin{abstract}
Transverse abdominis Plane blocks (TAP) provide effective postoperative analgesia following surgical incisions of the lower and middle abdominal wall, including those associated with cesarean section. This study investigated the efficacy of liposomal bupivacaine diluted with $0.25 \%$ bupivacaine administered in bilateral TAP blocks for post-operative analgesia after Cesarean section preformed under neuraxial anesthesia. The patients who received the TAP blocks with liposomal bupivacaine had noticeably low pain scores of 1.0 $\pm 1.4,1.4 \pm 2.1,1.7 \pm 1.9,1.9 \pm 3.3$ and $1.9 \pm 2.3$ at $6,12,24,48$ and 72 hours respectively. Only 3 patients used oxycodone $(5 \mathrm{mg}) /$ acetaminophen $(325 \mathrm{mg}$ ) postoperative. One patient took two tables of oxycodone $(5 \mathrm{mg}) /$ acetaminophen $(325 \mathrm{mg})$ after 24 hours, a second patient used oxycodone $(5 \mathrm{mg}) /$ acetaminophen $(325 \mathrm{mg}$ ) after 72 hours and the third patient was transferred to the intensive care unit (ICU) since she developed postpartum cardiac complications, and was give oxycodone ( $5 \mathrm{mg}$ )/acetaminophen $(325 \mathrm{mg}$ ) despite having a 0 pain score. These results suggest that patients treated bilateral TAP blocks with a mixture of liposomal and regular bupivacaine will have low pain scores, high patient satisfaction and reduce the use of postoperative narcotics.
\end{abstract}

\section{Keywords}

Liposomal Bupivacaine, Exparel, TAP Block, Cesarean-Section

\section{Introduction}

The Transversus Abdominis Plane (TAP) Block has been used to decrease postoperative pain since 2001 [1]. Multiple studies have demonstrated that TAP blocks provide effective postoperative analgesia for incisions on the mid and 
lower abdominal wall [2] [3] [4]. TAP blocks with non-liposomal ropivacaine and bupivacaine provide post-Cesarean section analgesia for up to $12-24$ hours [5] [6]. The post-partum minimization of intravenous opiate administration is especially important for nursing mothers who are concerned about opiate side effects on themselves and opiate levels in their breast milk [7]. There is limited data on the efficacy of liposomal bupivacaine in TAP blocks for control of postCesarean section pain. Exparel $^{\circledR}$, a liposomal bupivacaine, was approved in 2011 by the Food and Drug Administration. Exparel ${ }^{\circ}$ formulations combine bupivacaine with DepoFoam ${ }^{\oplus}$, a technology which delivers medication over an extended period of time. A single dose of liposomal bupivacaine in the transversus abdominis plane releases bupivacaine for up to 72 hours, thereby minimizing pain and the need for opiates throughout this time period. This objective of this study was to investigate the ability of liposomal bupivacaine, administered in bilateral TAP blocks, for lengthened post-operative analgesia after Cesarean sections and if the data demonstrated significant it would be used as the basis for future controlled, double blinded, and randomized study.

\section{Study Design}

This is a prospective case series of women who underwent an elective Cesarean delivery under spinal anesthesia. After institutional review board (IRB) approval, 12 participants at Maimonides Medical Center in Brooklyn, New York were consented to receive bilateral TAP blocks with liposomal bupivacaine for post Cesarean section analgesia and record their pain scores, usage of post-operative narcotics and overall satisfaction with their pain management. Each participant received an intraoperative spinal dose of hyperbaric bupivacaine $0.75 \%$, fentanyl $15 \mathrm{mcg}$, and preservative free morphine $0.2 \mathrm{mg}$, as per standard anesthetic practice for providing anesthesia for C-Section. Within minutes of incision closure, each participant received an ultrasound guided bilateral TAP block as previously described [8] with 20 milliliters of liposomal bupivacaine $(266 \mathrm{mg} / 20 \mathrm{ml} ; 13.3$ $\mathrm{mg} / \mathrm{ml}$ ); however, instead of diluting the liposomal bupivacaine with normal saline we diluted it with $20 \mathrm{ml}$ of $0.25 \%$ bupivacaine. The TAP plane was confirmed and created by separation of the transverses abdominis and internal oblique muscles with $5 \mathrm{ml}$ of $0.25 \%$ bupivacaine [8]. Subsequently, $20 \mathrm{ml}$ of the $0.25 \% /$ bupivacaine/ liposomal bupivacaine mixture was injected into this plane using real time ultrasound visualization. All subjects were ordered intravenous (IV) Tylenol and ketorolac as needed for mild pain and 1 - 2 tables of oxycodone $(5 \mathrm{mg}) /$ acetaminophen (325 mg) per os (p.o.) as needed for moderate to severe pain.

- The primary outcome was self-reported pain (using an 11-point (0 - 10) numeric rating scale (NRS) scale). From an earlier study [8] and from our increased use [9] [10] and application of liposomal bupivacaine [11], enrollment of 12 patients would allow us to investigate the ability of liposomal bupivacaine, administered in bilateral TAP blocks, for lengthened post-operative analgesia after Cesarean sections and give use the ability to plan a future 
double blinded randomized study.

- Secondary outcomes were reduction in the opiate use postoperatively and patient satisfaction.

- The incidence of post anesthesia adverse effects was also being noted.

\subsection{Inclusion Criteria}

- Post-partum women aged 18 - 45 years with American Society of Anesthesiology Class I-III physical status.

- All subjects undergoing elective Cesarean section with a spinal anesthetic.

- The subjects must be physically and mentally able to participate in the study and to complete all study assessments.

- The subjects must be able to give fully informed consent to participate in this study after demonstrating an understanding of the risks and benefits of the proposed components of the intervention (Bilateral TAP blocks).

\subsection{Exclusion Criteria}

- History of hypersensitivity or idiosyncratic reactions to amide-type local anesthetics.

- Any subject whose anatomy, or surgical procedure, in the opinion of the investigator, might preclude the potential successful completion of the bilateral TAP block.

- Any subject who in the opinion of the investigator, might be harmed or be a poor candidate for participation in the trial.

- Any subject, who in the opinion of the investigator, is on chronic opioid pain medications or regular use of large doses of nonsteroidal anti-inflammatory Drugs (NSAIDs).

- Subjects who have received any investigational drug within 30 days prior to study drug administration, or planned administration of another investigational product or procedure during their participation in the study.

- Subjects with history of psychiatric illnesses including previous postpartum depression.

\subsection{Efficacy Assessment}

- Subjects reported postsurgical pain after cesarean section using an 11-point (0 - 10) numeric rating scale (NRS) during the hospital stay. The pain scores were noted at $6,12,24,48$ and 72 hours postoperatively or until patient was discharged from the hospital if sooner than 72 hours.

- Date, time, amount and route of all opiate and non-opiate analgesics consumed by the patient.

- Subjects were asked about the overall satisfaction with post cesarean section analgesia at end of hospital stay and/or at the scheduled postoperative follow up visit (usually $10 \pm 5$ days after cesarean section). Inquiry was also made about the possible adverse events experienced since hospital discharge. 


\section{Results}

Patients demographic are listed in Table 1. Patients had an average age 31 years old. Their average weight was $82.5 \mathrm{kgs}$ with and average body mass index of 30.5. An average parity of 1.3 reflected a cohort of 3 primigravidas and 8 multigravidas. Only one patient lacked a high school diploma; whereas half of the patients had college degrees or graduate level education (Table 1).

Subjects who received bilateral TAP blocks with liposomal bupivacaine had resting pains scores, (mean \pm standard deviation (SD)) of $1.0 \pm 1.4,1.4 \pm 2.1,1.7$ $\pm 1.9,1.9 \pm 3.3$ and $1.9 \pm 2.3$ at $6,12,24,48$ and 72 hours respectively (Table 2). The mean pain score, when first requesting pain medication, was $1.3 \pm 2.3$ on a scale of 0 - 10. The mean pain score noted at all requests for pain medication was $0.8 \pm 1.8$. There were no reported adverse events.

Overall, patients in the TAP block group used little narcotics. Within 24 hours, only 1 patient used tables of oxycodone $(5 \mathrm{mg})$ /acetaminophen $(325 \mathrm{mg})$. On postoperative day 3, 72 hour after the TAP block, an additional patient requested and received $5 \mathrm{mgs}$ of oxycodone with $325 \mathrm{mgs}$ of acetaminophen 3 times. Additional, a third patient was transferred to the cardiac ICU for continuous monitoring since she had postpartum cardiomyopathy after her last pregnancy. She received tables of oxycodone $(5 \mathrm{mg}) /$ acetaminophen $(325 \mathrm{mg})$ every day for anticipated pain (even through her report pains were 0 ).

These results demonstrate that post Cesarean section patients receiving the TAP blocks with liposomal bupivacaine had significant postoperative pain relief (Table 2) and used little postoperative narcotic. Patient satisfaction scores were $9.3 \pm 1.1($ mean $\pm \mathrm{SD})($ Table 3$)$ on an 11 point scale.

\section{Discussion}

Intravenous and oral opiates have historically been the most commonly administered analgesics for post-Cesarean section pain. Osmundson et al. found in a prospective study that $83 \%$ of women used opiates for pain control for a median of 8 days post operatively after Cesarean delivery [12]. Bateman et al.'s analysis of Clinformatics Data Mart database found that $0.36 \%$ of opiate naïve women

Table 1. Characteristics of study population.

\begin{tabular}{cc}
\hline \multicolumn{1}{c}{ Patient Demographics } \\
\hline Age & $31 \pm 4.5$ \\
Weight kg & $82.1 \pm 16.7$ \\
Height CM & $163 \pm 7.0$ \\
BMI & $30.5 \pm 4.4$ \\
Parity & $1.3 \pm 1.0$ \\
Education & $2.6 \pm 0.9$
\end{tabular}

Patient characteristics: (Mean \pm Standard deviation, $\mathrm{N}=12$ ). Level of education was recorded: $1=\operatorname{Did}$ not graduate High School; 2 = High School Diploma; 3 = College Diploma; 4 = Graduate level education. 
Table 2. Patients pain scores after bilateral TAP Block after cesarean section.

\begin{tabular}{cccccccc}
\hline & 6 hours & 12 hours & 24 hours & 48 hours & 72 hours & Overall & \\
\hline Pain score & 1.0 & 1.4 & 1.7 & 1.9 & 1.9 & 1.5 & Mean \\
& 1.4 & 2.1 & 1.9 & 3.3 & 2.3 & 2.2 & SD \\
\hline
\end{tabular}

Patient pain scores from all 12 patients were recorded at every visit. There were asked on a scale of $0-10$, where 0 was no pain and 10 was the worst pain you can imagine: how do you rate you pain?

Table 3. Patient satisfaction with their pain management.

\begin{tabular}{cccccccc}
\hline & 6 hours & 12 hours & 24 hours & 48 hours & 72 hours & Overall & \\
\hline \multirow{2}{*}{ score } & 9.33 & 9.08 & 9.33 & 9.33 & 9.22 & 9.26 & Mean \\
& 1.07 & 1.24 & 1.15 & 0.78 & 1.30 & 1.07 & SD \\
\hline
\end{tabular}

Patient satisfaction were recorded from all 12 patients at either last visit or they were called at home. There were asked on a scale of $0-10$, where 0 was the worst and 10 was the best, how do you rate you pain management?

became persistent opiate users following Cesarean delivery [13]. Komatsu et al. analyzed opiate free functional recovery in normal vaginal delivery and Cesarean section and found that as many as $91 \%$ of women required opiates for postoperative pain with a median time for stopping opiates of 9 days (range of 0 - 39 days) [14]. Post-Cesarean section TAP blocks with liposomal bupivacaine likely decrease postoperative lengths of stay and opiate usage, thereby enabling decreases mothers to avoid opiate side effects such as sedation, hypoventilation, constipation, nausea, and vomiting as well as the potential for opiate addiction [15] [16] [17] [18]. Opiate sparing postoperative pain control allays the fear of mothers concerned with opiate delivered in breast milk.

TAP blocks with administration of non-liposomal local anesthetics offer minimal analgesic advantages, if any, over intrathecal or epidural opiates [19]. One randomized, double-blinded study by McMorrow et al. demonstrated that pain scores and IV opiate consumption were lowest in patients receiving intrathecal morphine with minimal improvement by the addition of TAP blocks with non-liposomal bupivacaine [20]. Similar findings have been observed in another meta-analysis but with additional opiate side effects in intrathecal morphine group [19]. Epidural morphine generally offers fewer risks as compared to parenteral opiate, but parenteral opiates are frequently required once the epidural catheter is removed [21].

The use of liposomal bupivacaine in TAP blocks decreases the potential for local anesthetic systemic toxicity in comparison with the non-liposomal form. The sustained DepoFoam lipid based delivery system in liposomal bupivacaine minimizes the likelihood of elevated local anesthetic plasm levels [22]. Griffiths et al. studied local anesthetic toxicity associated with TAP block using ropivacaine (2.5 mg per kilogram dose) and found that the total plasma concentration was elevated above the potential toxic threshold of 2.2 microgram per milliliters in the first hour post TAP blocks in 12 patients out of 30 . Three of these 12 pa- 
tients (25\%) reported symptoms of neuro toxicity (paresthesia, metallic taste and slurred speech) that resolved after 10 - 70 minutes [23]. Miranda et al. studied plasma absorption of levobupivacaine in TAP blocks and concluded that epinephrine should be added when levobupivacaine was used in doses $>1.5 \mathrm{mg}$ per kilogram in order to decrease toxicity symptoms by $0.1 \%$ [24].

This case series provides the only available prospective data on post-Cesarean section patients who received postoperative TAP blocks with liposomal bupivacaine. The primary weakness of this study lies in the small patient population and lack of randomization and blinding and lack of a controlled group. It also does not address both the variability in the dermatomal level of the administration of local anesthetics and the expertise of the anesthesiologist performing the injection. Further studies of liposomal bupivacaine in post cesarean section TAP blocks using a control group that will receive only regular bupivacaine in their bilateral TAP blocks is needed. This case series endorses the need for a larger double blinded randomized study with a control group, which could result in more powerful conclusions that would answer the question does liposomal bupivacaine significantly prolong the duration of bilateral TAP block after Caesarian section compared to regular bupivacaine, as well as, recovery room throughput, patient satisfaction/comfort, and record any adverse anesthesia effects.

\section{Conclusion}

The use of liposomal bupivacaine in TAP block provides long acting, post-Cesarean section analgesia. It enables patients to avoid or minimize opiate usage and addiction potential as well as their adverse side effects on both mother and newborn.

\section{References}

[1] Rafi, A.N. (2001) Abdominal Field Block: A New Approach via the Lumbar Triangle. Anaesthesia, 56, 1024-1026. https://doi.org/10.1046/j.1365-2044.2001.02279-40.x

[2] Belavy, D., Cowlishaw, P.J., Howes, M. and Phillips, F. (2009) Ultrasound-Guided Transversus Abdominis Plane Block for Analgesia after Caesarean Delivery. British Journal of Anaesthesia, 103, 726-730. https://doi.org/10.1093/bja/aep235

[3] Baaj, J.M., Alsatli, R.A., Majaj, H.A., Babay, Z.A. and Thallaj, A.K. (2010) Efficacy of Ultrasound-Guided Transversus Abdominis Plane (TAP) Block for Postcesarean Section Delivery Analgesia-A Double-Blind, Placebo-Controlled, Randomized Study. Middle East Journal of Anesthesiology, 20, 821-826.

[4] Carney, J., McDonnell, J.G., Ochana, A., Bhinder, R. and Laffey, J.G. (2008) The Transversus Abdominis Plane Block Provides Effective Postoperative Analgesia in Patients Undergoing Total Abdominal Hysterectomy. Anesthesia \& Analgesia, 107, 2056-2060. https://doi.org/10.1213/ane.0b013e3181871313

[5] Lee, A.J., Palte, H.D., Chehade, J.M., Arheart, K.L., Ranasinghe, J.S. and Penning, D.H. (2013) Ultrasound-Guided Bilateral Transversus Abdominis Plane Blocks in Conjunction with Intrathecal Morphine for Postcesarean Analgesia. Journal of 
Clinical Anesthesia, 25, 475-482. https://doi.org/10.1016/j.jclinane.2013.05.004

[6] Singh, S., Dhir, S., Marmai, K., Rehou, S., Silva, M. and Bradbury, C. (2013) Efficacy of Ultrasound-Guided Transversus Abdominis Plane Blocks for Post-Cesarean Delivery Analgesia: A Double-Blind, Dose-Comparison, Placebo-Controlled Randomized Trial. International Journal of Obstetric Anesthesia, 22, 188-193. https://doi.org/10.1016/j.ijoa.2013.03.003

[7] Beilin, Y., Bodian, C.A., Weiser, J., Hossain, S., Arnold, I., Feierman, D.E., et al. (2005) Effect of Labor Epidural Analgesia with and without Fentanyl on Infant Breast-Feeding: A Prospective, Randomized, Double-Blind Study. Anesthesiology, 103, 1211-1217. https://doi.org/10.1097/00000542-200512000-00016

[8] Feierman, D.E., Kronenfeld, M., Gupta, P.M., Younger, N. and Logvinskiy, E. (2014) Liposomal Bupivacaine Infiltration into the Transversus Abdominis Plane for Postsurgical Analgesia in Open Abdominal Umbilical Hernia Repair: Results from a Cohort of 13 Patients. Journal of Pain Research, 7, 477-482.

https://doi.org/10.2147/JPR.S65151

[9] Keilin, C., Salant, E.P., Andaz, C., Lawhon, R., Gupta, P. and Feierman, D.E. (2015) Use of Liposomal Bupivacaine (Exparel) for Reduction in the Use of Postoperative Narcotics in an Obese Patient Undergoing Modified Radical Mastectomy. Open Journal of Anesthesiology, 5.

[10] Garg, M., Gupta, P., Kang, K. and Feierman, A.D.D. (2017) Evaluation of the Efficacy of Liposomal Bupivacaine Infiltrated into the Posterior Capsule for Postoperative Analgesia after Total Knee Arthroplasty-A Randomized Double Blind Clinical Trial. Open Journal of Anesthesiology, 7.

[11] Lawhon, R., La Civita, R., Fanouse, J. and Feierman, D. (2015) Off Label Use of Exparel in an Axillary Block for Prolonged Postoperative Analgesia. Open Journal of Anesthesiology, 5.

[12] Osmundson, S.S., Schornack, L.A., Grasch, J.L., Zuckerwise, L.C., Young, J.L. and Richardson, M.G. (2017) Postdischarge Opioid Use after Cesarean Delivery. Obstetrics \& Gynecology, 130, 36-41. https://doi.org/10.1097/AOG.0000000000002095

[13] Bateman, B.T., Franklin, J.M., Bykov, K., Avorn, J., Shrank, W.H., Brennan, T.A., et al. (2016) Persistent Opioid Use Following Cesarean Delivery: Patterns and Predictors among Opioid-Naive Women. American Journal of Obstetrics \& Gynecology, 215, 353 e1-e18.

[14] Komatsu, R., Carvalho, B. and Flood, P.D. (2017) Recovery after Nulliparous Birth: A Detailed Analysis of Pain Analgesia and Recovery of Function. Anesthesiology, 127, 684-694. https://doi.org/10.1097/ALN.0000000000001789

[15] Fayezizadeh, M., Majumder, A., Neupane, R., Elliott, H.L. and Novitsky, Y.W. (2016) Efficacy of Transversus Abdominis Plane Block with Liposomal Bupivacaine during Open Abdominal Wall Reconstruction. The American Journal of Surgery, 212, 399-405. https://doi.org/10.1016/j.amjsurg.2015.12.026

[16] Gasanova, I., Alexander, J., Ogunnaike, B., Hamid, C., Rogers, D., Minhajuddin, A., et al. (2015) Transversus Abdominis Plane Block versus Surgical Site Infiltration for Pain Management after Open Total Abdominal Hysterectomy. Anesthesia \& Analgesia, 121, 1383-1388. https://doi.org/10.1213/ANE.0000000000000909

[17] Hutchins, J.L., Kesha, R., Blanco, F., Dunn, T. and Hochhalter, R. (2016) Ultrasound-Guided Subcostal Transversus Abdominis Plane Blocks with Liposomal Bupivacaine vs. Non-Liposomal Bupivacaine for Postoperative Pain Control after Laparoscopic Hand-Assisted Donor Nephrectomy: A Prospective Randomised Observer-Blinded Study. Anaesthesia, 71, 930-937. https://doi.org/10.1111/anae.13502 
[18] Hutchins, J., Delaney, D., Vogel, R.I., Ghebre, R.G., Downs Jr., L.S., Carson, L., et al. (2015) Ultrasound Guided Subcostal Transversus Abdominis Plane (TAP) Infiltration with Liposomal Bupivacaine for Patients Undergoing Robotic Assisted Hysterectomy: A Prospective Randomized Controlled Study. Gynecologic Oncology, 138, 609-613. https://doi.org/10.1016/j.ygyno.2015.06.008

[19] Abdallah, F.W., Halpern, S.H. and Margarido, C.B. (2012) Transversus Abdominis Plane Block for Postoperative Analgesia after Caesarean Delivery Performed under Spinal Anaesthesia? A Systematic Review and Meta-Analysis. British Journal of Anaesthesia, 109, 679-687. https://doi.org/10.1093/bja/aes279

[20] McMorrow, R.C., Ni Mhuircheartaigh, R.J., Ahmed, K.A., Aslani, A., Ng, S.C., Conrick-Martin, I., et al. (2011) Comparison of Transversus Abdominis Plane Block vs Spinal Morphine for Pain Relief after Caesarean Section. British Journal of Anaesthesia, 106, 706-712. https://doi.org/10.1093/bja/aer061

[21] Bonnet, M.P., Mignon, A., Mazoit, J.X., Ozier, Y. and Marret, E. (2010) Analgesic Efficacy and Adverse Effects of Epidural Morphine Compared to Parenteral Opioids after Elective Caesarean Section: A Systematic Review. European Journal of Pain, 14, 894 e1-e9.

[22] Golf, M., Daniels, S.E. and Onel, E. (2011) A Phase 3, Randomized, Placebo-Controlled Trial of DepoFoam (R) Bupivacaine (Extended-Release Bupivacaine Local Analgesic) in Bunionectomy. Advances in Therapy, 28, 776-788.

https://doi.org/10.1007/s12325-011-0052-y

[23] Griffiths, J.D., Le, N.V., Grant, S., Bjorksten, A., Hebbard, P. and Royse, C. (2013) Symptomatic Local Anaesthetic Toxicity and Plasma Ropivacaine Concentrations after Transversus Abdominis Plane Block for Caesarean Section. British Journal of Anaesthesia, 110, 996-1000. https://doi.org/10.1093/bja/aet015

[24] Miranda, P., Corvetto, M.A., Altermatt, F.R., Araneda, A., Echevarria, G.C. and Cortinez, L.I. (2016) Levobupivacaine Absorption Pharmacokinetics with and without Epinephrine during TAP Block: Analysis of Doses Based on the Associated Risk of Local Anaesthetic Toxicity. European Journal of Clinical Pharmacology, 72, 1221-1227. https://doi.org/10.1007/s00228-016-2086-1 